OPEN ACCESS

Edited by: Gianluca Tozzi, University of Portsmouth, United Kingdom

Reviewed by: Ralph Mueller,

ETH Zurich, Switzerland Brian Metscher University of Vienna, Austria

*Correspondence: Katherine A. Staines k.staines@napier.ac.uk

†Joint first authorship.

‡Joint senior authorship.

Specialty section: This article was submitted to Mechanics of Materials, a section of the journal

Frontiers in Materials

Received: 27 July 2017 Accepted: 22 December 2017 Published: 23 January 2018

Citation:

Staines KA, Madi K, Javaheri $B$, Lee $P D$ and Pitsillides AA (2018) A

Computed Microtomography Method for Understanding Epiphyseal Growth Plate Fusion.

Front. Mater. 4:48.

doi: 10.3389/fmats.2017.00048

\section{A Computed Microtomography Method for Understanding Epiphyseal Growth Plate Fusion}

\author{
Katherine A. Staines ${ }^{1 * t}$, Kamel Madi2t, Behzad Javaheri ${ }^{3}$, Peter D. Lee ${ }^{2 \ddagger}$ \\ and Andrew A. Pitsillides ${ }^{3+}$
}

'School of Applied Sciences, Edinburgh Napier University, Edinburgh, United Kingdom, ${ }^{2}$ School of Materials, The University of Manchester, Manchester, United Kingdom, ${ }^{3}$ Comparative Biomedical Sciences, Royal Veterinary College, London, United Kingdom

The epiphyseal growth plate is a developmental region responsible for linear bone growth, in which chondrocytes undertake a tightly regulated series of biological processes. Concomitant with the cessation of growth and sexual maturation, the human growth plate undergoes progressive narrowing, and ultimately disappears. Despite the crucial role of this growth plate fusion "bridging" event, the precise mechanisms by which it is governed are complex and yet to be established. Progress is hindered by the current methods for growth plate visualization; these are invasive and largely rely on histological procedures. Here, we describe our non-invasive method utilizing synchrotron X-ray computed microtomography for the examination of growth plate bridging, which ultimately leads to its closure coincident with termination of further longitudinal bone growth. We then apply this method to a dataset obtained from a benchtop micro computed tomography scanner to highlight its potential for wide usage. Furthermore, we conduct finite element modeling at the micron-scale to reveal the effects of growth plate bridging on local tissue mechanics. Employment of these 3D analyses of growth plate bone bridging is likely to advance our understanding of the physiological mechanisms that control growth plate fusion.

Keywords: growth plate, mouse, bone, synchrotron, computed tomography, fusion

\section{INTRODUCTION}

Endochondral ossification is a tightly regulated process responsible for the formation and postnatal linear growth of the long bones, including the tibia and femur. Endochondral ossification is carefully orchestrated to involve the replacement of a cartilage scaffold by mineralized bone, and integral to this is the epiphyseal growth plate, a developmental region located in the metaphysis of the long bones (Staines et al., 2012).

The growth plate consists of chondrocytes arranged in columns. These chondrocytes are surrounded by their extracellular matrix, consisting of specific collagens, namely Collagen types II and $\mathrm{X}$, as well as proteoglycans such as aggrecan and other non-collagenous matrix proteins including the SIBLING family of proteins (Ballock and O'Keefe, 2003; Mackie et al., 2011). Chondrocytes undergo distinct maturation stages of proliferation, differentiation, and hypertrophy, while maintaining their spatially fixed location (Hunziker et al., 1987). It is the terminally differentiated hypertrophic chondrocyte, which mineralizes its surrounding extracellular matrix (Castagnola et al., 1988). This 
process, thought to involve membrane-limited matrix vesicles, is biphasic and tightly regulated by a number of enzymes and factors including alkaline phosphatase (Alpl), PHOSPHO1, the ankylosis protein (Ank), ecto-nucleotide pyrophosphatase/phosphodiesterase-1 (Enpp1) (Terkeltaub et al., 1994; Anderson, 1995; Roberts et al., 2007). Mineralization of the cartilage extracellular matrix facilitates vascular invasion allowing the infiltration of bone resorbing osteoclasts and bone forming osteoblasts (Zelzer et al., 2002). This is a key step in endochondral ossification and enables the replacement of the cartilaginous scaffold by bone.

Throughout the majority of embryonic and postnatal longitudinal bone growth, the processes of mineralized cartilage production and replacement by bone are coupled. However, as growth slows, the human growth plate undergoes progressive narrowing as bony bridges form and span its width. This ultimately leads to complete growth plate closure and the cessation of human growth. These bone bridges are also known to form upon growth plate injury, thought to be through an intramembranous ossification mechanism (Xian et al., 2004). Despite this, the molecular mechanisms underpinning their formation are unknown. It is well established though that growth plate closure in humans coincides with sexual maturation with estrogen playing a pivotal role, although the precise mechanisms are complex and are also yet to be fully established. Indeed, in two genetic mutations resulting in estrogen deficiency (in the estrogen-receptor gene, and in the CYP19 gene), the growth plate fails to fuse and growth persists, albeit rather slowly, into adulthood (Smith et al., 1994; Morishima et al., 1995; Grumbach and Auchus, 1999; Grumbach, 2000).

Whether growth plate fusion occurs prior to or after the cessation of growth is of significant controversy in the field and has been somewhat overlooked (Parfitt, 2002). Evidence from studies in both humans and rats revealed the cessation of growth long before any histological evidence of growth plate fusion, suggesting that epiphyseal fusion is a marker of growth cessation and not its cause (Becks et al., 1948; Moss and Noback, 1958; Weise et al., 2001). This goes against the current state of knowledge and it is essential that the exact temporal and spatial relationship between growth cessation and growth plate fusion is delineated before the complexities of epiphyseal growth plate function can be fully understood.

Research into growth plate fusion mechanisms may have been hindered somewhat by the limitation in current methods available for its visualization, which are invasive and largely reliant upon histological procedures. While nano-scale imaging is now possible via many approaches, the high flux and superior detectors in synchrotron (SR) micro-computed tomography (CT) enable excellent spatial resolution and high speed imaging, ideal for the rapid collection of multiple image volumes. Furthermore, computational mechanobiology is recognized as powerful tool for predicting tissue growth and adaptation (Prendergast et al., 1997). The relationship between macroscopic mechanical loads and the local stresses and strains that influence tissue formation can now be calculated using computational models (Carter and Wong, 1988; Lacroix and Prendergast, 2002a,b; Lacroix et al., 2002; Shefelbine and Carter, 2004; Stokes et al., 2006; GarzónAlvarado et al., 2009; Gerhard et al., 2009; Lin et al., 2009; ReinaRomo et al., 2010; Schulte et al., 2013; Betts and Müller, 2014;
Giorgi et al., 2015). To the best of our knowledge, due to the lack of information on the undulating internal structure, the $3 \mathrm{D}$ morphology of the growth plate has not yet been included in models of endochondral ossification or its complex fusion mechanisms (Gao et al., 2014). It seems likely that the local mechanical stresses, which may contribute to such fusion will be better predicted by the addition of 3D morphology of the growth plate. Therefore, we have developed a readily accessible method that could be useful in discriminating between normal and abnormal growth plate dynamics.

Here, we describe a non-invasive method for the 3-dimensational (3D) quantification of growth plate bridging using gold standard SR microCT, which we have then also applied to images acquired using a standard, more widely accessible benchtop microCT scanner. We have then used these images to conduct finite element (FE) modeling to assess currently unexplored associations between growth plate bridging and local strain distributions. Exploitation of these new methods is likely to advance understanding of the physiological mechanisms, which lead to growth plate closure and the effects of this on local tissue mechanics.

\section{MATERIALS AND METHODS}

\section{Animal Model}

Wild-type mice (C57/BL6 and CBA) were housed up to four per cage in polypropylene cages with wood chip and paper bedding and provided standard mouse chow and water ad libitum throughout the study. Weaners up to 8 weeks of age were fed a standard rodent breeding diet and thereafter a standard rodent maintenance diet (Special Diet Services, South Witham, UK). All procedures complied with the UK Animals (Scientific Procedures) Act 1986 and were reviewed and approved by the ethics committee of the Royal Veterinary College (London, UK).

\section{Imaging}

\section{Tissue Imaging by Benchtop MicroCT}

The tibia from a 20-month-old female C57/BL6 mouse (Charles River, UK) was fixed in $70 \% \mathrm{EtOH}$ and stored until scanning using the Skyscan 1172 (Skyscan, Kontich, Belgium), with X-ray tube operated at $50 \mathrm{kV}$ and $200 \mu \mathrm{A}, 1,600 \mathrm{~ms}$ exposure time with a $0.5 \mathrm{~mm}$ aluminum filter and an acquisition pixel size of $5 \mu \mathrm{m}$. The slices were then reconstructed using NRecon 1.6.9.4 with a pixel matrix of 2,000 × 2,000 (Skyscan, Kontich, Belgium) (Javaheri et al., 2015).

\section{Tissue Imaging by SR MicroCT}

Tibiae from an 8- and 40-week old CBA mouse were dissected and frozen at $-20^{\circ} \mathrm{C}$ until scanning. The SR microCT was performed at Diamond Light Source on the Diamond-Manchester Branchline I13-2 using $19 \mathrm{keV}$ (based on an energy sensitivity study for optimum image contrast) monochromatic X-ray (Rau et al., 2011). 1,800 projections on $180^{\circ}$ were collected using a $4 \times$ magnification lens by a PCO 4000 CCD imaging camera with $4,008 \times 2,672$ pixels, giving an effective pixel size of $1.1 \mu \mathrm{m}$. The projections were normalized and reconstruction was performed 
with the tomography reconstruction module of the software Data Analysis WorkbeNch (Dawn) 1.7 (Basham et al., 2015), using Diamond's computing cluster to produce 3D volumes of the X-ray attenuation (Atwood et al., 2010; Titarenko et al., 2010). The images were filtered using a 3D median filter with a small kernel size of 2 to remove the "shot noise" common with tomography with minimal blurring effect on image texture.

\section{Development of a 3D Quantification Method for Growth Plate Bridging}

Tibiae scans from both benchtop microCT or SR microCT were used to identify growth plate bridges observed as indicated in Figure 1. Scans were segmented using $A_{v i z o}{ }^{\circledR}$ software (V8.0, VSG, Burlington, VT, USA), using a region-growing algorithm within the software. This algorithm starts from a seed point and selects all connected voxels with a gray value in a given tolerance interval. The volume images were aligned manually along the metaphyseal tibial shaft (defined as the $z$-axis; Figure 2A) and the central points of each individual bony bridge crossing the entire growth plate width were examined from slices cut in multiple orientations, and individually identified by an observer (Figure 2B). To reduce uncertainties due to partial volume effects, all the bridges smaller than about 125 voxels in volume were not considered. Once all the bridges were selected, each was quantified and rendered a separate color to confirm correct manual identification.

To quantify the bone bridge local number density, a method for projecting them onto the joint surface was developed. The center of all bridges (blue circles) were extracted using a skeletonization (Figure 2C) and each bridge was orthogonally projected onto the tibial joint surface using an in-house line intercept method implemented in Matlab (Mathworks, USA). The method consists of (1) generating straight lines along the $(z)$ direction passing through the bridges (Figure 2D), (2) determining the intersection between the straight lines and the tibial plateau (segmented using a region growing algorithm, Figure $2 \mathbf{E}$ ), and (3) detecting the endpoints of the lines that intercept the joint surface (Figure 2F). From this, the areal number density, $N$, is defined as the number of bridges per $256 \mu \mathrm{m} \times 256 \mu \mathrm{m}$ window (Figure 2G). The distribution of the areal number density of bridges is then superimposed on the tibial joint surface (each bridge has a color that represents the areal number density at the bridge location, Figure 2H) (Staines et al., 2016).

\section{FE Modeling}

The SR microCT images from the 8- and 40-week old mice were imported into the Avizo software (Avizo Fire, 9.2.0, VSG) to generate unstructured linear tetrahedral meshes using the methodology described by Madi et al. (2007). FE computations were carried out to simulate static compressive tests (sustained loading). The nodes at the bottom of the tibia have their vertical displacements fixed and certain rotations have been restrained to block rigid body motion. To restrain the rotations, one node is blocked in the three directions, one node is blocked along $X$ and one node is blocked along $Y$. At the top, loads equal to three times body weight $(\sim 1 \mathrm{~N})$ were applied as nodal forces (Cook et al., 1983 ) in the medial and lateral aspects of the tibia (Figure 3). Bone and growth plate cartilage tissues were treated as isotropic linear elastic materials (see Table 1) (Piszczatowski, 2012; Poulet et al., 2013). Following a mesh sensitivity study, the number of elements was fixed to about 2,500,000. The mesh density was approximately 250 voxels/element (the images were binned,
A

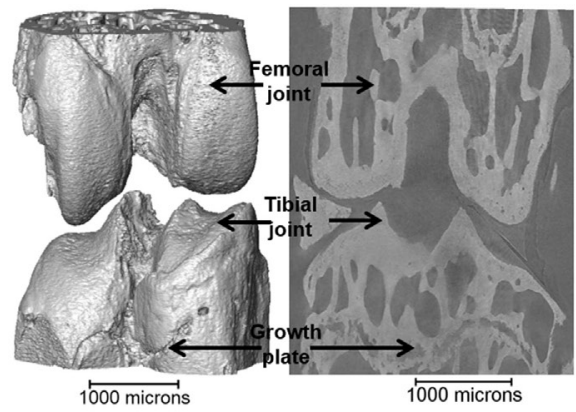

B

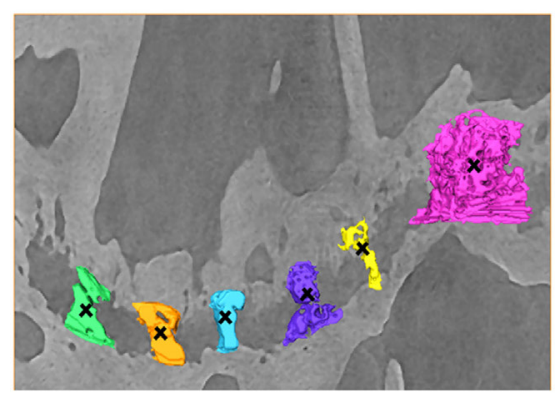

C

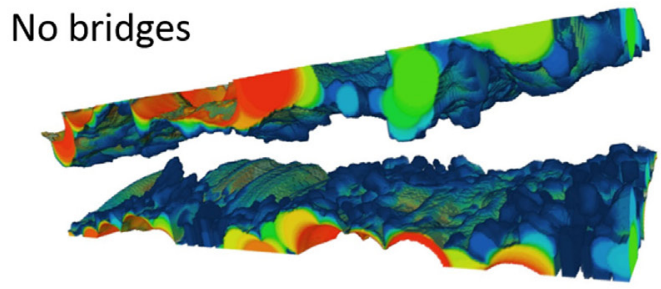

Bony bridges
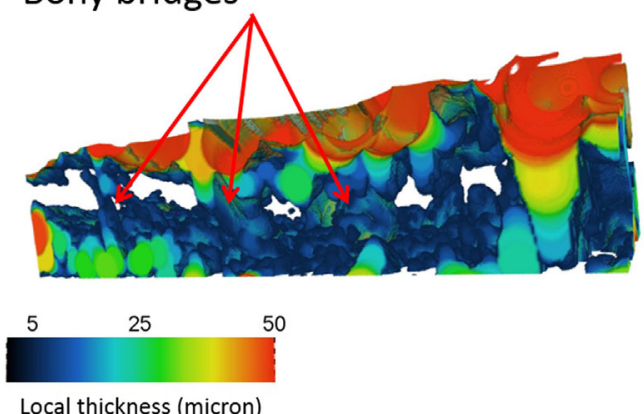

Local thickness (micron)

FIGURE 1 | Identification of the bridges crossing the growth plate (A) 3D representation of the whole joint, (B) bridges crossing the growth plate, in an ROI (black crosses indicate bony bridges identified by an observer). (C) 3D representation of the growth plate with no bridges and multiple bridges observed. Adapted from Staines et al. (2016). 


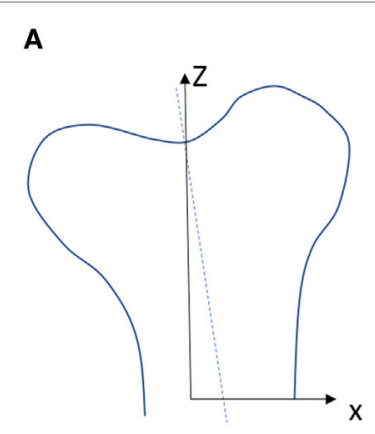

$E$

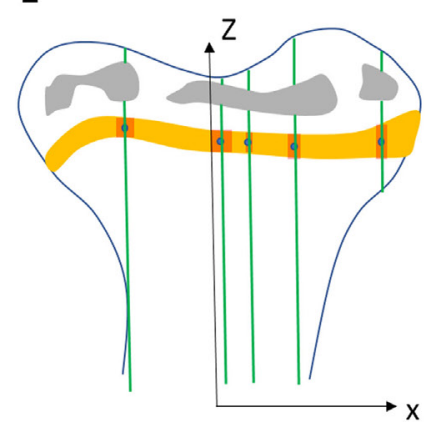

B

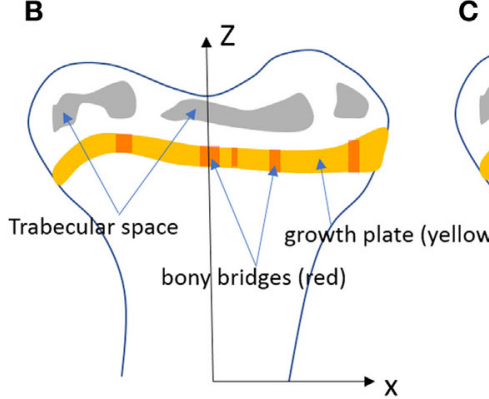

$\mathbf{F}$

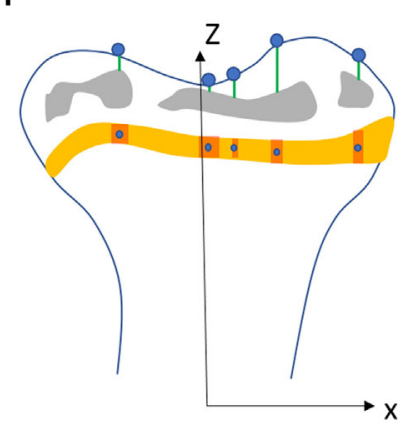

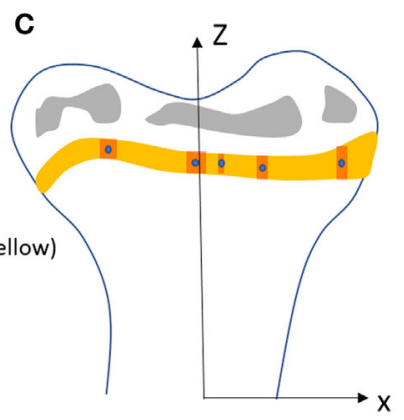

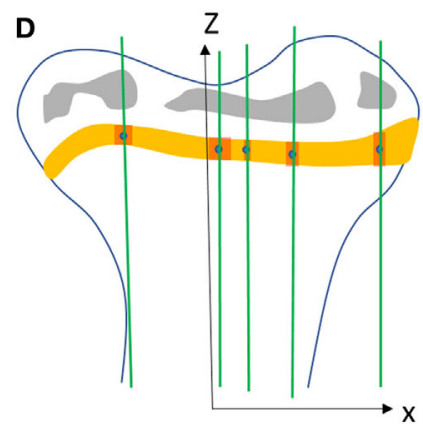

G

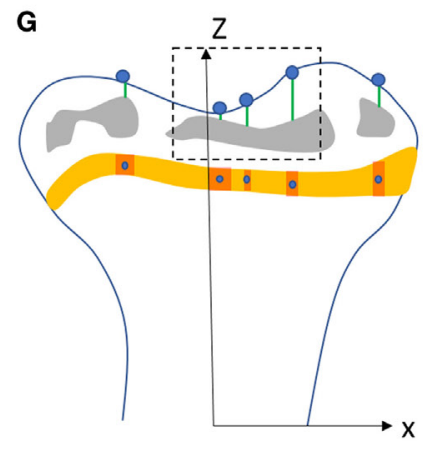

H

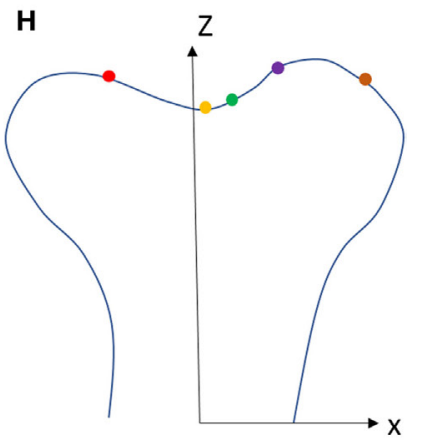

FIGURE 2 | Procedure developed to project the bridges and their areal number density onto the tibial joint surface: (A) the volume images were aligned along the metaphyseal tibial shaft (z-axis), (B) the central point of all bony bridges crossing the growth plate were examined from slices cut in multiple orientations, and identified by an observer who highlighted them individually with the cursor, (C) the center of all bridges (blue circles) were extracted using a skeletonization,

(D) generation of straight lines along the (Z) direction passing through the bridges, (E) intersection between the straight lines and the tibia (segmented using a region growing algorithm), (F) detection of endpoints of the lines that intercept the joint surface, (G) the areal number density, $N_{A}$, is calculated as the number of bridges per $256 \mu \mathrm{m} \times 256 \mu \mathrm{m}$ window, $\mathbf{( H )}$ the distribution of the areal number density of bridges is superimposed on the tibial joint surface (each bridge has a color that represents the areal number density at the bridge location).

i.e., $2.2 \mu \mathrm{m}$ voxel size), and was based on average and local (line profiles) axial stresses plotted against number of elements (error criterion: $5 \%$ of asymptotic value).

\section{RESULTS}

Herein, we used our method for the visualization and quantification of growth plate bridging, and our FE model to examine the effects of bridging on local tissue mechanics.

\section{Application of Novel Method to Benchtop MicroCT Scans}

We have previously shown that the above developed method is a valuable model for examining growth plate bridging during healthy and pathological aging (Staines et al., 2016). However, the use of SR microCT is not readily available for most laboratories due to high costs and availability of beamtime. Therefore we sought to examine whether our method could be applied to benchtop microCT at a pixel size of $5 \mu \mathrm{m}$. As detailed in our methods, the tibia from a C57/BL6 20-month-old female mouse was scanned using the SkyScan 1172 scanner. Images were reconstructed and our method for growth plate bridging 3D quantification method was applied (Figures 1 and 2). We were able to clearly identify growth plate bridges at $5 \mu \mathrm{m}$ and once all the bridges were selected, we were able to superimpose the distribution of the areal number density onto the tibial joint surface (Figure 4). Spatial evaluation of growth plate bridges indicates that thicker bridges are not randomly distributed but rather that these are preferentially enriched in peripheral growth plate locations, consistent with our previous results (Staines et al., 2016) (Figure 4).

\section{Application of 3D Quantification of Growth Plate Bridging in FE Models of the Loaded Tibia}

We reveal that in young ( 8 weeks old) CBA wild-type mice, growth plate bridging is associated with locations that contain high local von Mises stresses (Figures 5A,B). Moreover, we reveal that with aging an increased number and density of growth plate bridges is observed (Figure 5B), indicative of growth plate closure (Staines et al., 2016). Our FE modeling indicates that this increase in growth plate bridging with aging is translated into greater stresses in the growth plate (Figure 5C). This, therefore, offers insights into the biomechanical functionality of growth plate fusion.

\section{DISCUSSION}

The results presented in this study report a method for the $3 \mathrm{D}$ quantification of growth plate bridging in murine bones. Although 


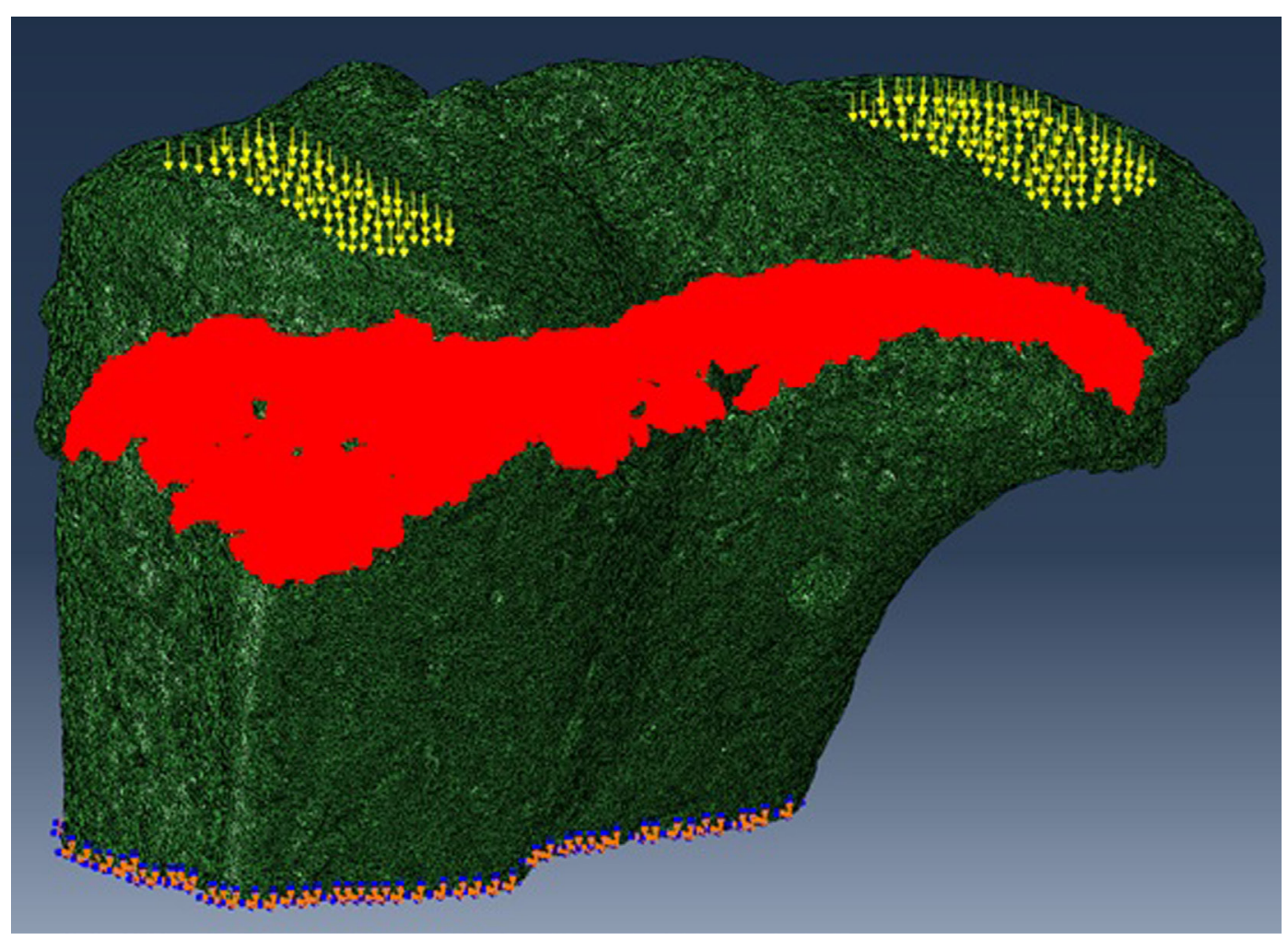

FIGURE 3 | For finite element (FE) modeling, FE computations were carried out to simulate static compressive tests (sustained loading). The nodes at the bottom of the tibia have their vertical displacements fixed and certain rotations are restrained to block rigid body motion. At the top, equal loads of three times body weight $(\sim 1 \mathrm{~N})$ were applied in the medial and lateral aspects of the tibia.

TABLE 1 | Material properties of bone and cartilage.

\begin{tabular}{lcc}
\hline Material properties & Bone & Growth plate \\
\hline$E(\mathrm{MPa})$ & 17,000 & 6 \\
Poisson coefficient & 0.3 & 0.49 \\
\hline
\end{tabular}

initially developed for SR microCT, we demonstrate that this method can also be applied to benchtop microCT scans collected at high resolutions. Furthermore, we reveal that this model can then be combined with FE modeling to understand local tissue mechanics. The relationship between mechanical loads and the local stresses and strains that influence tissue formation can be calculated using computational models for macro-, meso-, and nano-scales (Webster et al., 2008; Schulte et al., 2013). This method described herein will contribute to future advances in the development of hierarchical models for understanding bone development and adaptation.

We show that, in wild-type mice, increased growth plate bridging translates into increased stresses in the bone directly beneath the growth plate. At 8 weeks (Figure 5B), few bridges are detected and overall the growth plate is squeezed in a "sandwich" configuration. This suggests that compressive hydrostatic stresses are engendered across major volumes and that higher shear stresses are generated only at the peripheral edges of the growth plate. Yet, the results of numerous mechanobiological

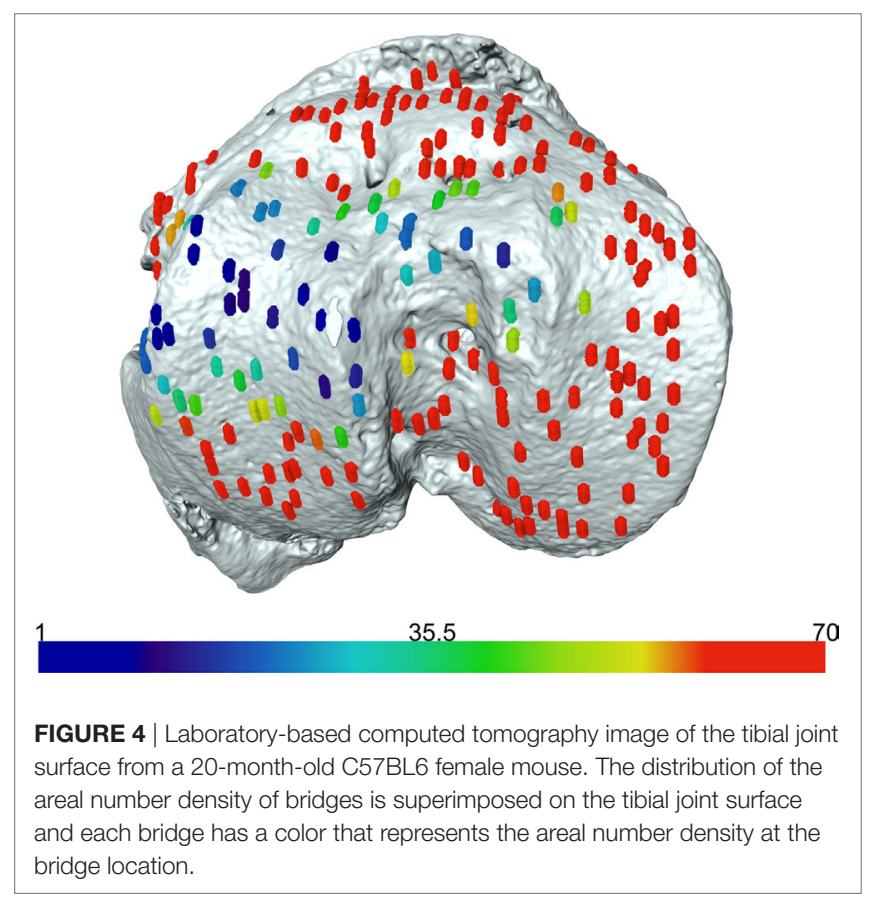

models support that growth and ossification is accelerated by tensile strain (or shear stresses) and that cartilage tends to be maintained by hydrostatic compressive stress (Carter and Wong, 


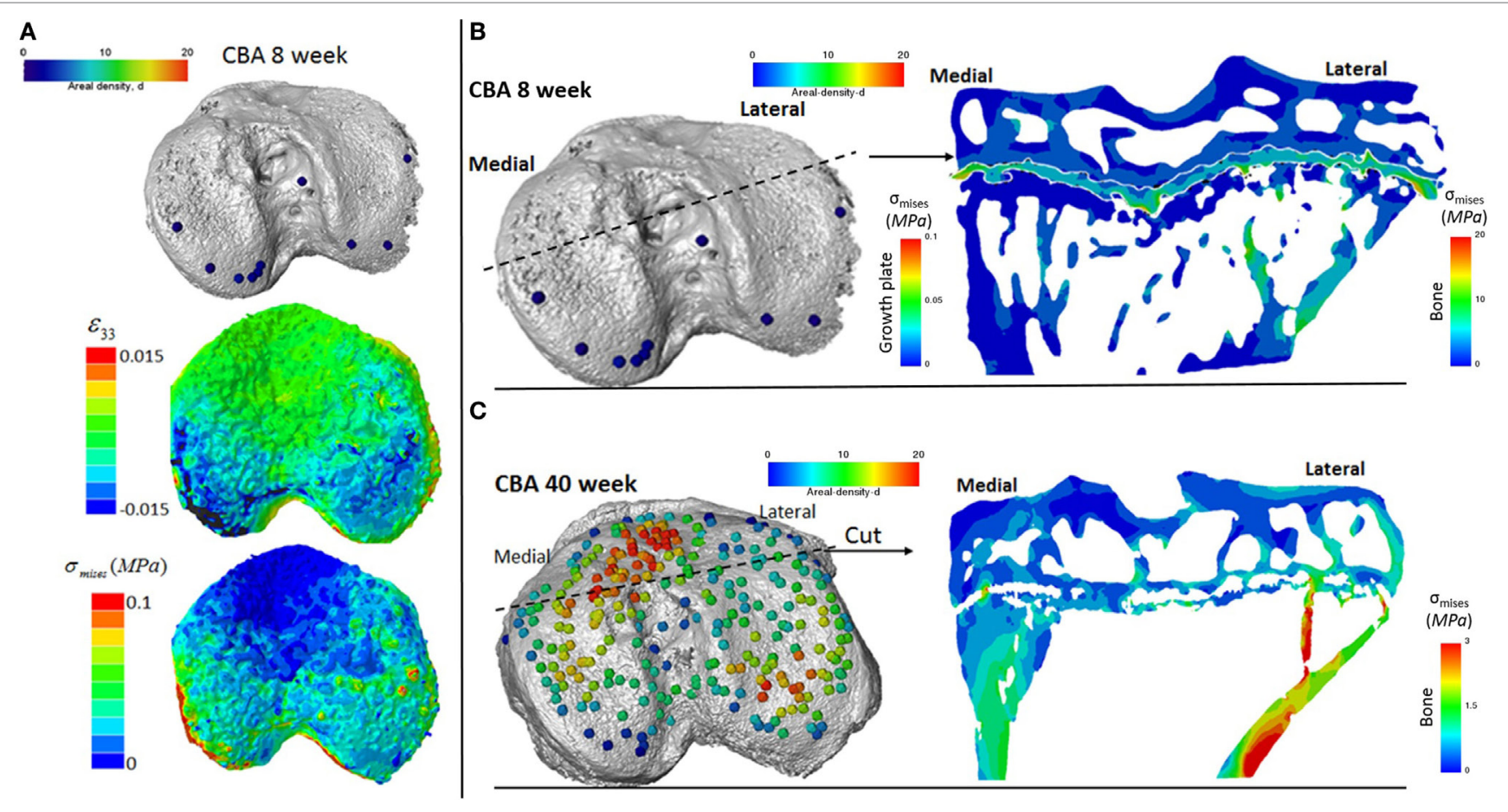

FIGURE 5 | Finite element models of the loaded tibia in CBA wild-type mice (A) visualization of von Mises stresses on the tibial joint plateau. Visualization of the stresses associated with growth plate bridging in a coronal section of the tibia for a (B) young (8 week) and (C) mature (40 week) mouse joint.

2003; Stokes et al., 2007; Villemure and Stokes, 2009). This would be consistent with our observations and may offer an explanation for why the bridges start growing first at the edges. At 40 weeks (Figure 5C), the mechanical environment of the growth plate is more complex; the thicker bridges act as stress concentrators. This appears to increase the likelihood that growth plate bridges in older animals will fracture under loading and/or achieve the redistribution of stresses to within their particular vicinities of the growth plate cartilage. Reciprocally, during endochondral ossification, growth-related strain in the mice may generate stresses that are contained by the bony bridges, accelerating growth arrest at these vicinities. More time points (between 8 and 40 weeks) and modeling are needed to support this hypothesis, but this work has provided novel insights into the use of $\mathrm{CT}$, advanced image processing, and FE modeling in understanding biomechanics in health and disease. Our methodology offers a unique mode for visualizing these events and the potential to address questions that have hitherto remained elusive in this field.

There are a number of unanswered questions in our pursuit of understanding growth plate closure such as; in which direction does growth plate bridging occur? Does bridging follow a conserved pattern across all growth plates? What is the trajectory of individual bridge expansion during growth cessation? And does genetics or mechanics define the initiation and progression of growth plate bridging? Such questions may now be addressable with the development of our novel non-invasive method for 3D quantification of bony bridging linked to FE models, and this will likely advance understanding of mechanisms involved in growth plate closure, which have hitherto been hindered somewhat by reliance upon invasive, largely histological methods.

The growth plate is responsible for the development and growth of long bones, up until puberty at which point it begins to close. During this closure, bone bridges form and span the width of the growth plate, eventually leading to the replacement of the entire growth plate cartilage anlagen by mineralized bone. Histological studies have identified this to be an early event involving several pre-osteoblastic molecules such as osteoprotegerin, interleukin-6, bone morphogenetic protein, and Collagen type X (Pichler et al., 2013). Similarly, trauma to the growth plate, e.g., in fractures, can also provoke abnormal growth plate bridging and the impairment of longitudinal growth.

Biomechanically, the growth plate is subject to a number of different loads placed upon it and previous studies have identified regional variations in the mechanical properties of the growth plate and surrounding tissues by confocal and atomic force microscopies (Bachrach et al., 1995; Radhakrishnan et al., 2004; Villemure et al., 2007). It is known that different compressive mechanical strains are found throughout the different zones of the growth plate, with the proliferative zone of chondrocytes exhibiting lower compressive strains than that of the resting and hypertrophic zones (Villemure et al., 2007). Similarly, compressive differences are also observed spatially throughout the growth plate with interior samples from bovine femoral growth plates being $40 \%$ more rigid than samples taken from the periphery of the growth plate (Cohen et al., 1994). Here, we reveal an association between growth plate bridging and increased stress 
dissipation distal to the growth plate, by applying FE modeling to our method. Future investigations will improve FE models to include more realistic boundary conditions, material properties, and cartilage mechanobiological principles. Our final aim is to examine the combined effects of hydrostatic pressure and shear stress (in the form of an osteogenix index) on the development of the skeleton and articular cartilage.

We have previously used SR microCT to study growth plate bridging in the context of a murine model of spontaneous osteoarthritis (Staines et al., 2016). Herein, we have also employed benchtop microCT to image the tibia of wild-type mice and confirm suitability of using benchtop microCT-based imaging for bridging analysis. Despite imaging at a lower resolution ( 5 instead of $1.1 \mu \mathrm{m}$ voxel size), we were able to replicate our method and confirm that in both imaging modalities, similar data can be obtained. Further, the data obtained from the benchtop microCT are analogous to the gold-standard SR imaging. Of course, there are disadvantages related to the use of dose radiation. This may have an impact on the mechanical properties of bone and cartilage and limit the validity of 4D in situ mechanical experiments as showed by Barth et al. (2010), but to our knowledge, exert little effect on the 3D morphology. The missing link in current mechanobiological models is the $3 \mathrm{D}$ real morphology of the growth plate which is very complex and cannot be captured correctly by $2 \mathrm{D}$ approaches. Herein, we have aimed to address this by describing and then utilizing our 3D non-destructive imaging methodology for the quantification of growth plate bridging. Employment of these 3D analyses of growth plate bone bridging is likely to advance our understanding of the physiological mechanisms, which lead to growth plate closure and subsequent FE modeling allows investigations into

\section{REFERENCES}

Anderson, H. C. (1995). Molecular biology of matrix vesicles. Clin. Orthop. Relat. Res. 314, 266-280.

Atwood, R. C., Lee, P. D., Konerding, M. A., Rockett, P., and Mitchell, C. A. (2010). Quantitation of microcomputed tomography-imaged ocular microvasculature. Microcirculation 17, 59-68. doi:10.1111/j.1549-8719.2009.00009.x

Bachrach, N. M., Valhmu, W. B., Stazzone, E., Ratcliffe, A., Lai, W. M., and Mow, V. C. (1995). Changes in proteoglycan synthesis of chondrocytes in articular cartilage are associated with the time-dependent changes in their mechanical environment. J. Biomech. 28, 1561-1569. doi:10.1016/0021-9290(95) 00103-4

Ballock, R. T., and O'Keefe, R. J. (2003). Physiology and pathophysiology of the growth plate. Birth Defects Res. C Embryo Today 69, 123-143. doi:10.1002/ bdrc. 10014

Barth, H. D., Zimmermann, E. A., Schaible, E., Tang, S. Y., Alliston, T., and Ritchie, R. O. (2010). Characterization of the effects of $x$-ray irradiation on the hierarchical structure and mechanical properties of human cortical bone. Biomaterials 32, 8892-8904. doi:10.1016/j.biomaterials.2011. 08.013

Basham, M., Filik, J., Wharmby, M. T., Chang, P. C., El Kassaby, B., Gerring, M., et al. (2015). Data Analysis WorkbeNch (DAWN). J Synchrotron Radiat 22, 853-858. doi:10.1107/S1600577515002283

Becks, H., Asling, C. W., Collins, D. A., Simpson, M. E., and Evans, H. M. (1948). Changes with increasing age in the ossification of the third metacarpal of the female rat. Anat. Rec. 100, 577-591. doi:10.1002/ar.1091000407

Betts, D. C., and Müller, R. (2014). Mechanical regulation of bone regeneration: theories, models, and experiments. Front. Endocrinol. 2014:5. doi:10.3389/ fendo.2014.00211 the associated biomechanical functionality. This will ultimately enable investigators to study the role of local tissue mechanics on endochondral ossification patterns, skeletal morphology, and articular cartilage function.

\section{ETHICS STATEMENT}

This study was carried out in accordance with the recommendations of the UK Animals (Scientific Procedures) Act 1986 and were reviewed and approved by the ethics committee of the Royal Veterinary College (London, UK).

\section{AUTHOR CONTRIBUTIONS}

Conception and design of work: KS, KM, PL, and AP. Acquisition of data: KM and BJ. Interpretation of data, revising the manuscript and final approval, and agreement to be accountable for all aspects of the work: KS, KM, BJ, PL, and AP. Drafting the manuscript: KS.

\section{FUNDING}

This work was funded by Arthritis Research UK (18768 and 20581) and by the EPSRC (EP/I02249X/1). Facilities and research support were provided by the Diamond-Manchester Branchline (I13-2) at Diamond Light Source (Beamtimes MT13237-1, MT11076-1, MT5003-1). The datasets generated during and/ or analysed during this study are not publicly available due to their large size but are available from the corresponding author on reasonable request.

Carter, D. R., and Wong, M. (1988). Mechanical stresses and endochondral ossification in the chondroepiphysis. J. Orthop. Res. 6, 148-154. doi:10.1002/ jor. 1100060120

Carter, D. R., and Wong, M. (2003). Modelling cartilage mechanobiology. Philos. Trans. R. Soc. Lond. B Biol. Sci. 358. Available at: http://rstb.royalsocietypublishing.org/content/358/1437/1461

Castagnola, P., Dozin, B., Moro, G., and Cancedda, R. (1988). Changes in the expression of collagen genes show two stages in chondrocyte differentiation in vitro. J. Cell Biol. 106, 461-467. doi:10.1083/jcb.106.2.461

Cohen, B., Chorney, G. S., Phillips, D. P., Dick, H. M., and Mow, V. C. (1994). Compressive stress-relaxation behavior of bovine growth plate may be described by the nonlinear biphasic theory. J. Orthop. Res. 12, 804-813. doi:10.1002/ jor. 1100120608

Cook, S. D., Lavernia, C. J., Burke, S. W., Skinner, H. B., and Haddad, R. J. Jr. (1983). A biomechanical analysis of the etiology of the tibia vara. J. Pediatr. Orthop. 3, 449-454. doi:10.1097/01241398-198309000-00006

Gao, J., Williams, J. L., and Roan, E. (2014). On the state of stress in the growth plate under physiologic compressive loading. Open J. Biophys. 4, 41661-41670. doi:10.4236/ojbiphy.2014.41003

Garzón-Alvarado, D. A., García-Aznar, J. M., and Doblaré, M. (2009). A reaction-diffusion model for long bones growth. Biomech. Model. Mechanobiol. 8, 381-395. doi:10.1007/s10237-008-0144-Z

Gerhard, F. A., Webster, D. J., van Lenthe, G. H., and Müller, R. (2009). In silico biology of bone modelling and remodelling: adaptation. Philos. Trans. A Math. Phys. Eng. Sci. 367, 2011-2030. doi:10.1098/rsta.2008.0297

Giorgi, M., Carriero, A., Shefelbine, S. J., and Nowlan, N. C. (2015). Effects of normal and abnormal loading conditions on morphogenesis of the prenatal hip joint: application to hip dysplasia. J. Biomech. 2015, 3390-3397. doi:10.1016/j. jbiomech.2015.06.002 
Grumbach, M. M. (2000). Estrogen, bone, growth and sex: a sea change in conventional wisdom. J. Pediatr. Endocrinol. Metab. 13(Suppl. 6), 1439-1455. doi:10.1515/jpem-2000-s619

Grumbach, M. M., and Auchus, R. J. (1999). Estrogen: consequences and implications of human mutations in synthesis and action. J. Clin. Endocrinol. Metab. 84, 4677-4694. doi:10.1210/jcem.84.12.6290

Hunziker, E. B., Schenk, R. K., and Cruz-Orive, L. M. (1987). Quantitation of chondrocyte performance in growth-plate cartilage during longitudinal bone growth. J. Bone Joint Surg. Am. 69, 162-173. doi:10.2106/00004623-19876902000002

Javaheri, B., Carriero, A., Staines, K. A., Chang, Y. M., Houston, D. A., Oldknow, K. J., et al. (2015). Phospho1 deficiency transiently modifies bone architecture yet produces consistent modification in osteocyte differentiation and vascular porosity with ageing. Bone 81, 277-291. doi:10.1016/j.bone.2015.07.035

Lacroix, D., and Prendergast, P. J. (2002a). A mechano-regulation model for tissue differentiation during fracture healing: analysis of gap size and loading. J. Biomech. 35, 1163-1171. doi:10.1016/S0021-9290(02)00086-6

Lacroix, D., and Prendergast, P. J. (2002b). Three-dimensional simulation of fracture repair in the human tibia. Comput. Methods Biomech. Biomed. Engin. 2002, 369-376. doi:10.1080/1025584021000025014

Lacroix, D., Prendergast, P. J., Li, G., and Marsh, D. (2002). Biomechanical model to simulate tissue differentiation and bone regeneration: application to fracture healing. Med. Biol. Eng. Comput. 2002, 14-21. doi:10.1007/BF02347690

Lin, H., Aubin, C. E., Parent, S., and Villemure, I. (2009). Mechanobiological bone growth: comparative analysis of two biomechanical modeling approaches. Med. Biol. Eng. Comput. 47, 357-366. doi:10.1007/s11517-008-0425-9

Mackie, E. J., Tatarczuch, L., and Mirams, M. (2011). The skeleton: a multifunctional complex organ: the growth plate chondrocyte and endochondral ossification. J. Endocrinol. 211, 109-121. doi:10.1530/JOE-11-0048

Madi, K., Forest, S., Boussuge, M., Gailliègue, S., Lataste, E., Buffière, J., et al. (2007). Finite element simulations of the deformation of fused-cast refractories based on X-ray computed tomography. Comput. Mater. Sci. 39, 224-229. doi:10.1016/j.commatsci.2006.01.033

Morishima, A., Grumbach, M. M., Simpson, E. R., Fisher, C., and Qin, K. (1995). Aromatase deficiency in male and female siblings caused by a novel mutation and the physiological role of estrogens. J. Clin. Endocrinol. Metab. 80, 3689-3698. doi:10.1210/jcem.80.12.8530621

Moss, M. L., and Noback, C. R. (1958). A longitudinal study of digital epiphyseal fusion in adolescence. Anat. Rec. 131, 19-32. doi:10.1002/ar.1091310103

Parfitt, A. M. (2002). Misconceptions (1): epiphyseal fusion causes cessation of growth. Bone 30, 337-339. doi:10.1016/S8756-3282(01)00668-8

Pichler, K., Musumeci, G., Vielgut, I., Martinelli, E., Sadoghi, P., Loreto, C., et al. (2013). Towards a better understanding of bone bridge formation in the growth plate? An immunohistochemical approach. Connect. Tissue Res. 54, 408-415. d oi:10.3109/03008207.2013.828715

Piszczatowski, S. (2012). Geometrical aspects of growth plate modelling using Carters and Stokess approaches. Acta Bioeng. Biomech. 14, 93-106.

Poulet, B., Westerhof, T. A., Hamilton, R. W., Shefelbine, S. J., and Pitsillides, A. A. (2013). Spontaneous osteoarthritis in Str/ort mice is unlikely due to greater vulnerability to mechanical trauma. Osteoarthr. Cartil. 21, 756-763. doi:10.1016/j. joca.2013.02.652

Prendergast, P. J., Huiskes, R., and Søballe, K. (1997). Biophysical stimuli on cells during tissue differentiation at implant interfaces. J. Biomech. 30, 539-548. doi:10.1016/S0021-9290(96)00140-6

Radhakrishnan, P., Lewis, N. T., and Mao, J. J. (2004). Zone-specific micromechanical properties of the extracellular matrices of growth plate cartilage. Ann. Biomed. Eng. 32, 284-291. doi:10.1023/B:ABME.0000012748.41851.b4

Rau, C., Wagner, U., Pesic, Z., and De Fanis, A. (2011). Coherent imaging at the diamond beamline I13. Phys. Status Solidi 208, 2522-2525. doi:10.1002/ pssa.201184272

Reina-Romo, E., Gómez-Benito, M. J., García-Aznar, J. M., Domínguez, J., and Doblaré, M. (2010). Growth mixture model of distraction osteogenesis: effect of pre-traction stresses. Biomech. Model. Mechanobiol. 2010, 103-115. doi:10.1007/s10237-009-0162-5
Roberts, S., Narisawa, S., Harmey, D., Millán, J. L., and Farquharson, C. (2007). Functional involvement of PHOSPHO1 in matrix vesicle-mediated skeletal mineralization. J. Bone Miner. Res. 22, 617-627. doi:10.1359/jbmr.070108

Schulte, F. A., Ruffoni, D., Lambers, F. M., Christen, D., Webster, D. J., Kuhn, G., et al. (2013). Local mechanical stimuli regulate bone formation and resorption in mice at the tissue level. PLoS ONE 2013:e62172. doi:10.1371/journal.pone.0062172

Shefelbine, S. J., and Carter, D. R. (2004). Mechanobiological predictions of growth front morphology in developmental hip dysplasia. J. Orthop. Res. 22, 346-352. doi:10.1016/j.orthres.2003.08.004

Smith, E. P., Boyd, J., Frank, G. R., Takahashi, H., Cohen, R. M., Specker, B., et al. (1994). Estrogen resistance caused by a mutation in the estrogen-receptor gene in a man. N. Engl. J. Med. 331, 1056-1061. doi:10.1056/NEJM199410203311604

Staines, K. A., Macrae, V. E., and Farquharson, C. (2012). The importance of the SIBLING family of proteins on skeletal mineralisation and bone remodelling. J. Endocrinol. 214, 241-255. doi:10.1530/JOE-12-0143

Staines, K. A., Madi, K., Mirczuk, S. M., Parker, S., Burleigh, A., Poulet, B., et al. (2016). Endochondral growth defect and deployment of transient chondrocyte behaviors underlie osteoarthritis onset in a natural murine model. Arthritis Rheumatol. 68, 880-891. doi:10.1002/art.39508

Stokes, I. A., Aronsson, D. D., Dimock, A. N., Cortright, V., and Beck, S. (2006). Endochondral growth in growth plates of three species at two anatomical locations modulated by mechanical compression and tension. J. Orthop. Res. 24, 1327-1334. doi:10.1002/jor.20189

Stokes, I. A., Clark, K. C., Farnum, C. E., and Aronsson, D. D. (2007). Alterations in the growth plate associated with growth modulation by sustained compression or distraction. Bone 41, 197-205. doi:10.1016/j.bone.2007.04.180

Terkeltaub, R., Rosenbach, M., Fong, F., and Goding, J. (1994). Causal link between nucleotide pyrophosphohydrolase overactivity and increased intracellular inorganic pyrophosphate generation demonstrated by transfection of cultured fibroblasts and osteoblasts with plasma cell membrane glycoprotein-1. Relevance to cal. Arthritis Rheum. 37, 934-941. doi:10.1002/art.1780370624

Titarenko, V., Titarenko, S., Withers, P. J., De Carlo, F., and Xiao, X. (2010). Improved tomographic reconstructions using adaptive time-dependent intensity normalization. J. Synchrotron Radiat. 17, 689-699. doi:10.1107/S0909049510024908

Villemure, I., Cloutier, L., Matyas, J. R., and Duncan, N. A. (2007). Non-uniform strain distribution within rat cartilaginous growth plate under uniaxial compression. J. Biomech. 40, 149-156. doi:10.1016/j.jbiomech.2005.11.008

Villemure, I., and Stokes, I. A. F. (2009). Growth plate mechanics and mechanobiology. A survey of present understanding. J. Biomech. 45, 1793-1803. doi:10.1016/j.jbiomech.2009.05.021

Webster, D. J., Morley, P. L., van Lenthe, G. H., and Müller, R. (2008). A novel in vivo mouse model for mechanically stimulated bone adaptaion - a combined experimental and compulational validation study. Comput. Methods Biomech. Biomed. Eng. 11, 435-441. doi:10.1080/10255840802078014

Weise, M., De-Levi, S., Barnes, K. M., Gafni, R. I., Abad, V., and Baron, J. (2001). Effects of estrogen on growth plate senescence and epiphyseal fusion. Proc. Natl. Acad. Sci. U.S.A. 98, 6871-6876. doi:10.1073/pnas. 121180498

Xian, C. J., Zhou, F. H., McCarty, R. C., and Foster, B. K. (2004). Intramembranous ossification mechanism for bone bridge formation at the growth plate cartilage injury site. J. Orthop. Res. 22, 417-426. doi:10.1016/j.orthres.2003.08.003

Zelzer, E., McLean, W., Ng, Y. S., Fukai, N., Reginato, A. M., Lovejoy, S., et al. (2002). Skeletal defects in VEGF(120/120) mice reveal multiple roles for VEGF in skeletogenesis. Development 129, 1893-1904.

Conflict of Interest Statement: The authors declare that the research was conducted in the absence of any commercial or financial relationships that could be construed as a potential conflict of interest.

Copyright $\odot 2018$ Staines, Madi, Javaheri, Lee and Pitsillides. This is an open-access article distributed under the terms of the Creative Commons Attribution License (CC BY). The use, distribution or reproduction in other forums is permitted, provided the original author(s) or licensor are credited and that the original publication in this journal is cited, in accordance with accepted academic practice. No use, distribution or reproduction is permitted which does not comply with these terms. 